\title{
STABILITY AND CONVERGENCE OF SECOND-ORDER SCHEMES FOR THE NONLINEAR EPITAXIAL GROWTH MODEL WITHOUT SLOPE SELECTION
}

\author{
ZHONGHUA QIAO, ZHI-ZHONG SUN, AND ZHENGRU ZHANG
}

\begin{abstract}
We present one nonlinear and one linearized numerical schemes for the nonlinear epitaxial growth model without slope selection. Both schemes are proved to be uniquely solvable and convergent with the convergence rate of order two in a discrete $L_{2}$-norm. By introducing an auxiliary variable in the discrete energy functional, the energy stability of both schemes is guaranteed regardless of the time step size, in the sense that a modified energy is monotonically nonincreasing in discrete time. Numerical experiments are carried out to support the theoretical claims.
\end{abstract}

\section{INTRODUCTION}

We consider the nonlinear two-dimensional fourth-order PDE designed to model epitaxial growth of thin films without slope selection [6, 9]:

$$
\frac{\partial u}{\partial t}+\delta \Delta^{2} u+\nabla \cdot\left(\frac{\nabla u}{1+|\nabla u|^{2}}\right)=0, \quad(x, y) \in \Omega, 0<t \leq T,
$$

subject to the initial value condition

$$
u(x, y, 0)=u_{0}(x, y), \quad(x, y) \in \bar{\Omega},
$$

and the periodic boundary conditions. Here $u=u(x, y, t)$ is the scaled height function of a thin film in a co-moving frame, $\delta$ is a constant, and $\Omega=[a, b] \times[c, d]$. Problems of this type model coarsening process arising in various fields such as chemistry, physics, ecology and biology [5, 20. The fourth-order term models surface diffusion, and the nonlinear second-order term models the Ehrlich-Schwoebel effect. This growth equation (1.1) defines the gradient flow with respect to the energy functional rendered by the $L^{2}(\Omega)$ inner product; viz.

$$
E(u)=\iint_{\Omega}\left(\frac{\delta}{2}|\Delta u|^{2}-\frac{1}{2} \ln \left(1+|\nabla u|^{2}\right)\right) d x d y .
$$

The second term in the energy $-\frac{1}{2} \ln \left(1+|\nabla u|^{2}\right)$ is bounded above but unbounded below. Furthermore, it has no relative minima, which implies that there are no

Received by the editor October 13, 2012 and, in revised form, June 4, 2013.

2010 Mathematics Subject Classification. Primary 65M06, 65M12, 65Z05.

Key words and phrases. Molecular beam epitaxy, finite difference scheme, energy decay, stability, convergence, linearized difference scheme.

The research of the first author was partially supported by the Hong Kong RGC grant PolyU 2021/12P and the Hong Kong Polytechnic University grants A-PL61 and 1-ZV9Y.

The second author was supported by NSFC under Grant 11271068.

The research of the third author was supported by NSFC under Grants 11071124, 11271048, 91130021 and the Fundamental Research Funds for the Central Universities. 
energetically favored values for $|\nabla u|$. At the physical point of view, this means that there is no slope selection mechanism in the epitaxial growth dynamics. A detailed discussion and additional references may be found in [2,6, 16]. The wellposedness of a suitable initial-boundary-value problem involving (1.1) was studied by $\mathrm{Li}$ and Liu [15] using perturbation analysis. Two energy identities were also given.

It has been predicted that mound structures in the surface profile will be generated in the coarsening process and will grow unbounded 13. Some mathematical justification of the coarsening scaling laws and mound growth rate has been produced. See [14,16] and the references therein. However, it is difficult to analyze the growth detail of the mound structures, especially for complex thin-film systems, so numerical simulation has played an important role in investigating the evolution process of thin-film epitaxial growth at large times. Most existing continuum model simulations have used the explicit Euler algorithm in time and a finite difference approximation in space, when the number of spatial grid points must be large and the time step quite small to maintain numerical stability and to achieve high accuracy. Even with rapidly increasing computing resources, explicit schemes remain limited to simulating only the early surface evolution of thin-film epitaxial growth [15]. Implicit treatment of the fourth-order term in (1.1) may be expected to relax the time step restriction [18, but numerical experiments have demonstrated that a larger time step cannot be used when $\delta$ is small; e.g. see [15. To improve this, energy stable schemes have attracted considerable attention recently, where the energy stability is guaranteed regardless of the time step size, in the sense that the energy is monotonically nonincreasing in discrete time. Some conditionally energy stable linear splitting schemes were suggested in 27] for the molecular beam epitaxial (MBE) model with slope selection and in 11] for the Cahn-Hilliard equation. Splitting parameters are involved, which should be chosen sufficiently large to ensure the energy stability. However, these parameters depend on the unknown solutions. A convex splitting of the energy functional method was exploited by Eyre [7, to study the unconditionally energy stable time discretization of the Cahn-Hilliard equation. Eyre's idea was generalized to the case of equations for thin-film epitaxy where the energy functional was decomposed into convex and concave parts, with the convex part treated implicitly and the concave part explicitly in the time discretization [25]. Two nonlinear schemes that are first-order in time were proposed for thin-film epitaxy, to solve with or without slope selection. These schemes are unconditionally uniquely solvable and unconditionally energy stable. Besides application to the energy functional (1.3), the convex splitting method has been used to solve Swift-Hohenberg and phase field crystal type equations [12, 26. Du and Nicolades proposed a different energy stable scheme for the Cahn-Hilliard equation involving a finite element discretization in space [4, and a finite difference version was subsequently analyzed by Furihata for one space dimension [8] . His scheme has also been used to study the thin-film epitaxial model in 19. with an adaptive time-stepping strategy.

It is challenging but important to construct energy stable higher order schemes and linear schemes for the thin-film epitaxy model (1.1). Recently, a linear firstorder in time scheme was developed in 2 and two second-order in time schemes were constructed for thin-film epitaxy [22, using the method of convex splitting of the energy functional. In this paper, we first construct a nonlinear Crank-Nicolson type 
finite difference scheme. Then by linearizing the nonlinear term, we obtain a linear four-level scheme. Both schemes are proved to be uniquely solvable and of secondorder convergence in a discrete $L_{2}$-norm. By introducing an auxiliary variable in the energy functional and discretizing its associated equation in a proper manner, the energy stability of both schemes is guaranteed based on a modified energy regardless of the time step size. A similar approach was presented in 10 for solving the CahnHilliard equation, where the auxiliary variable $q(x, t)$ was introduced, which is not a Lagrange multiplier but allows us to enforce the sphere condition $|\phi|=1$. By using this approach, a linear unconditional stable and uniquely solvable scheme was obtained for solving the Cahn-Hilliard equation. In [10], an equation related to the auxiliary variable $q(x, t)$ needs to be solved together with the mixed formulation of the Cahn-Hilliard equation for updating the unknown function $\phi$. However, in our approach, the discrete equations related to the auxiliary variable is solved only for updating the modified energies.

A brief outline of the paper is as follows. Definitions and basic notations are given in Section 2. An energy stable nonlinear second-order scheme with the stability and convergence analysis is presented in Section 3, followed by a description of an energy stable fully linear second-order scheme in Section 4. In Section 5 , the results of numerical experiments with test problems from the literature are presented, and comparisons are made with other published solution techniques. Concluding remarks are given in Section 6.

\section{Preliminaries}

Let

$$
v=\ln \left(1+|\nabla u|^{2}\right)
$$

then

$$
\frac{\partial v}{\partial t}=\frac{\left(|\nabla u|^{2}\right)_{t}}{1+|\nabla u|^{2}}=\frac{2 \nabla u \cdot \nabla u_{t}}{1+|\nabla u|^{2}},
$$

and the energy functional (1.3) can be written as

$$
E(u)=\iint_{\Omega} \frac{1}{2}\left(\delta|\Delta u|^{2}-v(x, y, t)\right) d x d y=\frac{1}{2}\left(\delta\|\Delta u(\cdot, \cdot, t)\|^{2}-(v(\cdot, \cdot, t), 1)\right) .
$$

Lemma 2.1 (Existence, uniqueness and energy identity [15]). Let $u_{0} \in H_{\text {per }}^{2}(\Omega)$. Then, the initial-boundary-value problem (1.1)-(1.2) has a unique solution $u: \Omega \times$ $[0, T] \rightarrow R$ that satisfies the following energy identities:

$$
\begin{aligned}
& \frac{d}{d t}\|u\|^{2}+4 E(u)+2 \iint_{\Omega}\left(\frac{1}{1+|\nabla u|^{2}}+\ln \left(1+|\nabla u|^{2}\right)\right) d x d y=2|\Omega|, \\
& \frac{d}{d t} E(u)+\left\|\frac{\partial u}{\partial t}\right\|^{2}=0
\end{aligned}
$$

where $|\Omega|$ is the area of the domain.

Let $h_{1}=(b-a) / M_{1}, \quad h_{2}=(d-c) / M_{2}, \quad \Delta t=T / N, \quad x_{i}=a+i h_{1}, \quad y_{j}=$ $c+j h_{2}, \quad t_{n}=n \Delta t$. Denote

$$
\begin{gathered}
\Omega_{h}=\left\{\left(x_{i}, y_{j}\right) \mid 0 \leq i \leq M_{1}, 0 \leq j \leq M_{2}\right\}, \quad \Omega_{\tau}=\left\{t_{n} \mid 0 \leq n \leq N\right\}, \\
\mathcal{V}_{h}=\left\{u \mid u=\left\{u_{i j}\right\}, u_{i+M_{1}, j}=u_{i j}, u_{i, j+M_{2}}=u_{i j}\right\} .
\end{gathered}
$$


For $u \in \mathcal{V}_{h}$, denote

$$
\begin{aligned}
& \delta_{x} u_{i+\frac{1}{2}, j}=\frac{1}{h_{1}}\left(u_{i+1, j}-u_{i, j}\right), \quad \delta_{y} u_{i, j+\frac{1}{2}}=\frac{1}{h_{2}}\left(u_{i, j+1}-u_{i, j}\right), \\
& \Delta_{x} u_{i j}=\frac{1}{2 h_{1}}\left(u_{i+1, j}-u_{i-1, j}\right), \quad \Delta_{y} u_{i j}=\frac{1}{2 h_{2}}\left(u_{i, j+1}-u_{i, j-1}\right), \\
& \delta_{x}^{2} u_{i j}=\frac{1}{h_{1}^{2}}\left(u_{i+1, j}-2 u_{i j}+u_{i-1, j}\right), \quad \delta_{y}^{2} u_{i j}=\frac{1}{h_{2}^{2}}\left(u_{i, j+1}-2 u_{i j}+u_{i, j-1}\right), \\
& \nabla_{h} u_{i j}=\left(\Delta_{x} u_{i j}, \Delta_{y} u_{i j}\right)^{T}, \quad \Delta_{h} u_{i j}=\left(\delta_{x}^{2}+\delta_{y}^{2}\right) u_{i j} .
\end{aligned}
$$

It is obvious that

$$
\Delta_{x} u_{i j}=\frac{1}{2}\left(\delta_{x} u_{i-\frac{1}{2}, j}+\delta_{x} u_{i+\frac{1}{2}, j}\right), \quad \Delta_{y} u_{i j}=\frac{1}{2}\left(\delta_{y} u_{i, j-\frac{1}{2}}+\delta_{y} u_{i, j+\frac{1}{2}}\right)
$$

and

$$
\delta_{x}^{2} u_{i j}=\frac{1}{h_{1}}\left(\delta_{x} u_{i+\frac{1}{2}, j}-\delta_{x} u_{i-\frac{1}{2}, j}\right), \quad \delta_{y}^{2} u_{i j}=\frac{1}{h_{2}}\left(\delta_{y} u_{i, j+\frac{1}{2}}-\delta_{y} u_{i, j-\frac{1}{2}}\right) .
$$

For a grid function $w=\left(w^{0}, w^{1}, \cdots, w^{N-1}, w^{N}\right)$ on $\Omega_{\tau}$, denote

$$
\begin{array}{ll}
w^{n+\frac{1}{2}}=\frac{1}{2}\left(w^{n}+w^{n+1}\right), & 0 \leq n \leq N-1 \\
\hat{w}^{n+\frac{1}{2}}=2 w^{n-\frac{1}{2}}-w^{n-\frac{3}{2}}, & 2 \leq n \leq N-1 .
\end{array}
$$

It is easy to know that

$$
\hat{w}^{n+\frac{1}{2}}=w^{n}+\frac{1}{2} w^{n-1}-\frac{1}{2} w^{n-2}, \quad 2 \leq n \leq N-1 .
$$

For $u \in \mathcal{V}_{h}$ and $v \in \mathcal{V}_{h}$, define the inner product

$$
(u, v)=h_{1} h_{2} \sum_{i=1}^{M_{1}} \sum_{j=1}^{M_{2}} u_{i j} \cdot v_{i j}
$$

and Sobolev norms (or seminorms)

$$
\begin{aligned}
& \|u\|=\sqrt{(u, u)}, \quad\|u\|_{\infty}=_{1 \leq i \leq M_{1}, 1 \leq j \leq M_{2}}\left|u_{i j}\right|, \\
& \left\|\delta_{x} u\right\|=\sqrt{h_{1} h_{2} \sum_{i=1}^{M_{1}} \sum_{j=1}^{M_{2}}\left|\delta_{x} u_{i-\frac{1}{2}, j}\right|^{2}}, \quad\left\|\delta_{y} u\right\|=\sqrt{h_{1} h_{2} \sum_{i=1}^{M_{1}} \sum_{j=1}^{M_{2}}\left|\delta_{y} u_{i, j-\frac{1}{2}}\right|^{2}} \\
& \left\|\nabla_{h} u\right\|=\sqrt{h_{1} h_{2} \sum_{i=1}^{M_{1}} \sum_{j=1}^{M_{2}}\left|\nabla_{h} u_{i j}\right|^{2}}, \quad\left\|\Delta_{h} u\right\|=\sqrt{h_{1} h_{2} \sum_{i=1}^{M_{1}} \sum_{j=1}^{M_{2}}\left|\Delta_{h} u_{i j}\right|^{2}}
\end{aligned}
$$

Lemma 2.2. For any grid function $u \in \mathcal{V}_{h}$, we have [21,29]

$$
\left\|\nabla_{h} u\right\|^{2} \leq\|u\| \cdot\left\|\Delta_{h} u\right\|
$$

\section{Nonlinear two-LEvel CRANK-Nicolson type sCheme}

Define the grid functions $U^{n} \in \mathcal{V}_{h}, V^{n} \in \mathcal{V}_{h}$ as follows:

$$
U_{i j}^{n}=u\left(x_{i}, y_{j}, t_{n}\right), \quad V_{i j}^{n}=v\left(x_{i}, y_{j}\right) .
$$


Then according to (1.1) and (2.2) with the Taylor expansion, we have

$$
\begin{array}{r}
\frac{U_{i j}^{n+1}-U_{i j}^{n}}{\Delta t}+\delta \Delta_{h}^{2} U_{i j}^{n+\frac{1}{2}}+\nabla_{h} \cdot\left(\frac{\nabla_{h} U_{i j}^{n+\frac{1}{2}}}{1+\left|\nabla_{h} U_{i j}^{n+\frac{1}{2}}\right|^{2}}\right)=R_{i j}^{n}, \\
1 \leq i \leq M_{1}, 1 \leq j \leq M_{2}, 0 \leq n \leq N-1
\end{array}
$$

and

$$
\begin{aligned}
\frac{V_{i j}^{n+1}-V_{i j}^{n}}{\Delta t}= & \frac{2 \nabla_{h} U_{i j}^{n+\frac{1}{2}} \cdot \nabla_{h} \frac{U_{i j}^{n+1}-U_{i j}^{n}}{\Delta t}}{1+\left|\nabla_{h} U_{i j}^{n+\frac{1}{2}}\right|^{2}}+S_{i j}^{n}, \\
& 1 \leq i \leq M_{1}, 1 \leq j \leq M_{2}, 0 \leq n \leq N-1,
\end{aligned}
$$

where there exists a constant $c_{1}$ such that

$$
\begin{aligned}
& \left|R_{i j}^{n}\right| \leq c_{1}\left(h_{1}^{2}+h_{2}^{2}+\Delta t^{2}\right), \quad 1 \leq i \leq M_{1}, \quad 1 \leq j \leq M_{2}, \quad 0 \leq n \leq N-1, \\
& \left|S_{i j}^{n}\right| \leq c_{1}\left(h_{1}^{2}+h_{2}^{2}+\Delta t^{2}\right), \quad 1 \leq i \leq M_{1}, \quad 1 \leq j \leq M_{2}, \quad 0 \leq n \leq N-1
\end{aligned}
$$

noticing the initial conditions

$$
U_{i j}^{0}=u_{0}\left(x_{i}, y_{j}\right), \quad V_{i j}^{0}=\ln \left(1+\left|\nabla_{h} U_{i j}^{0}\right|^{2}\right), \quad 1 \leq i \leq M_{1}, \quad 1 \leq j \leq M_{2} .
$$

Omitting the small terms in (3.1) and (3.2), we construct the difference scheme for (1.1)-(1.2) as follows:

Finding $u^{n} \in \mathcal{V}_{h}, v^{n} \in \mathcal{V}_{h}(0 \leq n \leq N)$ such that

$$
\begin{gathered}
\frac{u_{i j}^{n+1}-u_{i j}^{n}}{\Delta t}+\delta \Delta_{h}^{2} u_{i j}^{n+\frac{1}{2}}+\nabla_{h} \cdot\left(\frac{\nabla_{h} u_{i j}^{n+\frac{1}{2}}}{1+\left|\nabla_{h} u_{i j}^{n+\frac{1}{2}}\right|^{2}}\right)=0, \\
1 \leq i \leq M_{1}, 1 \leq j \leq M_{2}, 0 \leq n \leq N-1, \\
u_{i j}^{0}=u_{0}\left(x_{i}, y_{j}\right), \quad 1 \leq i \leq M_{1}, \quad 1 \leq j \leq M_{2},
\end{gathered}
$$

and

$$
\begin{aligned}
& \frac{v_{i j}^{n+1}-v_{i j}^{n}}{\Delta t}=\frac{2 \nabla_{h} u_{i j}^{n+\frac{1}{2}} \cdot \nabla_{h} \frac{u_{i j}^{n+1}-u_{i j}^{n}}{\Delta t}}{1+\left|\nabla_{h} u_{i j}^{n+\frac{1}{2}}\right|^{2}}, \\
& \quad 1 \leq i \leq M_{1}, 1 \leq j \leq M_{2}, 0 \leq n \leq N-1 . \\
& v_{i j}^{0}=\ln \left(1+\left|\nabla_{h} u_{i j}^{0}\right|^{2}\right) .
\end{aligned}
$$

3.1. The stability. The difference scheme (3.6)-(3.8) is unconditionally energy stable in the sense that a modified energy is monotonically nonincreasing in discrete time.

Theorem 3.1. Let $u^{n}$ and $v^{n}$ be the solution of difference scheme (3.6)-(3.8) and denote

$$
E_{h}^{n}=\frac{1}{2}\left[\delta\left\|\triangle_{h} u^{n}\right\|^{2}-\left(v^{n}, 1\right)\right], \quad 0 \leq n \leq N .
$$


Then, we have

$$
\left\|\frac{u^{n+1}-u^{n}}{\Delta t}\right\|^{2}+\frac{E_{h}^{n+1}-E_{h}^{n}}{\Delta t}=0, \quad 0 \leq n \leq N-1 .
$$

Proof. Taking the inner product of (3.6) with $\frac{u^{n+1}-u^{n}}{\Delta t}$ leads to

$$
\begin{aligned}
& \left\|\frac{u^{n+1}-u^{n}}{\Delta t}\right\|^{2}+\delta\left(\Delta_{h}^{2} u^{n+\frac{1}{2}}, \frac{u^{n+1}-u^{n}}{\Delta t}\right) \\
& \quad+\left(\nabla_{h} \cdot\left(\frac{\nabla_{h} u^{n+\frac{1}{2}}}{1+\left|\nabla_{h} u^{n+\frac{1}{2}}\right|^{2}}\right), \frac{u^{n+1}-u^{n}}{\Delta t}\right)=0 .
\end{aligned}
$$

For the middle term on the left-hand of (3.10), we have

$$
\begin{aligned}
\left(\Delta_{h}^{2} u^{n+\frac{1}{2}}, \frac{u^{n+1}-u^{n}}{\Delta t}\right) & =\left(\Delta_{h} u^{n+\frac{1}{2}}, \Delta_{h} \frac{u^{n+1}-u^{n}}{\Delta t}\right) \\
& =\frac{\delta}{2 \Delta t}\left(\left\|\Delta_{h} u^{n+1}\right\|^{2}-\left\|\Delta_{h} u^{n}\right\|^{2}\right) .
\end{aligned}
$$

For the last term on the left-hand of (3.10), we have

$$
\begin{aligned}
\left(\nabla_{h}\right. & \left.\cdot\left(\frac{\nabla_{h} u^{n+\frac{1}{2}}}{1+\left|\nabla_{h} u^{n+\frac{1}{2}}\right|^{2}}\right), \frac{u^{n+1}-u^{n}}{\Delta t}\right) \\
& =-\left(\frac{\nabla_{h} u^{n+\frac{1}{2}}}{1+\left|\nabla_{h} u^{n+\frac{1}{2}}\right|^{2}}, \nabla_{h} \frac{u^{n+1}-u^{n}}{\Delta t}\right) \\
& =-\frac{1}{2 \Delta t}\left(v^{n+1}-v^{n}, 1\right) .
\end{aligned}
$$

Inserting (3.11) and (3.12) into (3.10) yields

$$
\begin{aligned}
\left\|\frac{u^{n+1}-u^{n}}{\Delta t}\right\|^{2}+\frac{1}{\Delta t}\left[\frac{1}{2}(\right. & \left.\delta\left\|\Delta_{h} u^{n+1}\right\|^{2}-\left(v^{n+1}, 1\right)\right) \\
\left.-\frac{1}{2}\left(\delta\left\|\Delta_{h} u^{n}\right\|^{2}-\left(v^{n}, 1\right)\right)\right] & =0
\end{aligned}
$$

or

$$
\left\|\frac{u^{n+1}-u^{n}}{\Delta t}\right\|^{2}+\frac{E_{h}^{n+1}-E_{h}^{n}}{\Delta t}=0, \quad 0 \leq n \leq N-1 .
$$

This completes the proof.

3.2. The solvability. We shall use the following Brouwer fixed-point theorem to show the existence of solution of the Crank-Nicolson type finite difference scheme (3.6) - (3.7)

Lemma $3.2([1,24])$. Let $(H,(\cdot, \cdot))$ be a finite dimensional inner product space, $\|\cdot\|$ the associated norm, and let $g: H \rightarrow H$ be continuous. Assume, moreover, that

$$
\exists \alpha>0, \forall z \in H,\|z\|=\alpha,(g(z), z) \geq 0 .
$$

Then, there exists an element $z^{*} \in H$ such that $g\left(z^{*}\right)=0$ and $\left\|z^{*}\right\| \leq \alpha$. 
Theorem 3.3. The difference scheme (3.6)-(3.7) has at least one solution.

Proof. The difference scheme (3.6) can be written as

$\frac{u_{i j}^{n+\frac{1}{2}}-u_{i j}^{n}}{\Delta t / 2}+\delta \Delta_{h}^{2} u_{i j}^{n+\frac{1}{2}}+\nabla_{h} \cdot\left(\frac{\nabla_{h} u_{i j}^{n+\frac{1}{2}}}{1+\left|\nabla_{h} u_{i j}^{n+\frac{1}{2}}\right|^{2}}\right)=0, \quad 1 \leq i \leq M_{1}, \quad 1 \leq j \leq M_{2}$

or

$$
\begin{aligned}
& w_{i j}-u_{i j}^{n}+\frac{\Delta t}{2} \delta \Delta_{h}^{2} w_{i j}+\frac{\Delta t}{2} \nabla_{h} \cdot\left(\frac{\nabla_{h} w_{i j}}{1+\left|\nabla_{h} w_{i j}\right|^{2}}\right)=0, \\
& 1 \leq i \leq M_{1}, \quad 1 \leq j \leq M_{2},
\end{aligned}
$$

with $w_{i j}=u_{i j}^{n+\frac{1}{2}}$.

Define the map

$$
\begin{aligned}
& g(w)_{i j}=w_{i j}-u_{i j}^{n}+\frac{\Delta t}{2} \delta \Delta_{h}^{2} w_{i j}+\frac{\Delta t}{2} \nabla_{h} \cdot(\left.\frac{\nabla_{h} w_{i j}}{1+\left|\nabla_{h} w_{i j}\right|^{2}}\right)=0, \\
& 1 \leq i \leq M_{1}, \quad 1 \leq j \leq M_{2} .
\end{aligned}
$$

Then

$$
\begin{aligned}
& (g(w), w) \\
& \quad=(w, w)-\left(w, u^{n}\right)+\frac{\Delta t}{2} \delta\left(\Delta_{h}^{2} w, w\right)+\frac{\Delta t}{2}\left(\nabla_{h} \cdot\left(\frac{\nabla_{h} w}{1+\left|\nabla_{h} w\right|^{2}}\right), w\right) \\
& \quad=(w, w)-\left(w, u^{n}\right)+\frac{\Delta t}{2} \delta\left\|\Delta_{h} w\right\|^{2}-\frac{\Delta t}{2}\left(\frac{\nabla_{h} w}{1+\left|\nabla_{h} w\right|^{2}}, \nabla_{h} w\right) \\
& \quad \geq\|w\|^{2}-\|w\| \cdot\left\|u^{n}\right\|-\frac{\Delta t}{2}|\Omega| \\
& \quad \geq \frac{1}{2}\left(\|w\|^{2}-\left\|u^{n}\right\|^{2}-|\Omega|\right) .
\end{aligned}
$$

Take $\alpha=\left\|u^{n}\right\|^{2}+|\Omega|$. Then if $w \in \mathcal{V}_{h}$ and $\|w\|=\alpha$, we have $(g(w), w) \geq 0$. By Lemma 3.2 , there is at least one solution $w$ satisfying $\|w\| \leq\left\|u^{n}\right\|^{2}+|\Omega|$. This completes the proof.

Now we consider the uniqueness. We need the following lemma.

\section{Lemma 3.4.}

$$
\begin{aligned}
2\left(\left|\nabla_{h} z\right|^{2} \nabla_{h} w-\left|\nabla_{h} w\right|^{2} \nabla_{h} z\right) & =\left(\left|\nabla_{h} z\right|^{2}+\left|\nabla_{h} w\right|^{2}\right) \nabla_{h}(w-z) \\
& -\left[\left(\nabla_{h} w+\nabla_{h} z\right) \cdot \nabla_{h}(w-z)\right]\left(\nabla_{h} w+\nabla_{h} z\right) .
\end{aligned}
$$

Proof. Adding

$$
\begin{aligned}
& \left|\nabla_{h} z\right|^{2} \nabla_{h} w-\left|\nabla_{h} w\right|^{2} \nabla_{h} z \\
& \quad=\left|\nabla_{h} z\right|^{2}\left(\nabla_{h} w-\nabla_{h} z\right)-\left(\left|\nabla_{h} w\right|^{2}-\left|\nabla_{h} z\right|^{2}\right) \nabla_{h} z \\
& \quad=\left|\nabla_{h} z\right|^{2}\left(\nabla_{h} w-\nabla_{h} z\right)-\left[\left(\nabla_{h} w+\nabla_{h} z\right) \cdot\left(\nabla_{h} w-\nabla_{h} z\right)\right] \nabla_{h} z
\end{aligned}
$$


and

$$
\begin{aligned}
& \left|\nabla_{h} z\right|^{2} \nabla_{h} w-\left|\nabla_{h} w\right|^{2} \nabla_{h} z \\
& \quad=\left|\nabla_{h} w\right|^{2}\left(\nabla_{h} w-\nabla_{h} z\right)-\left(\left|\nabla_{h} w\right|^{2}-\left|\nabla_{h} z\right|^{2}\right) \nabla_{h} w \\
& \quad=\left|\nabla_{h} w\right|^{2}\left(\nabla_{h} w-\nabla_{h} z\right)-\left[\left(\nabla_{h} w+\nabla_{h} z\right) \cdot\left(\nabla_{h} w-\nabla_{h} z\right)\right] \nabla_{h} w,
\end{aligned}
$$

we get the equality required. This completes the proof.

Theorem 3.5. If $\Delta t<32 \delta / 9$, then the difference scheme (3.6)-(3.7) has at most one solution.

Proof. Suppose that (3.14) has an another solution $z_{i j}$, which satisfies

$$
\begin{aligned}
& z_{i j}-u_{i j}^{n}+\frac{\Delta t}{2} \delta \Delta_{h}^{2} z_{i j}+\frac{\Delta t}{2} \nabla_{h} \cdot\left(\frac{\nabla_{h} z_{i j}}{1+\left|\nabla_{h} z_{i j}\right|^{2}}\right)=0, \\
& 1 \leq i \leq M_{1}, \quad 1 \leq j \leq M_{2} .
\end{aligned}
$$

Let

$$
\epsilon_{i j}=w_{i j}-z_{i j}, \quad 1 \leq i \leq M_{1}, \quad 1 \leq j \leq M_{2} .
$$

Subtracting (3.15) from (3.14), we have

$$
\begin{aligned}
& \epsilon_{i j}+\frac{\Delta t}{2} \delta \Delta_{h}^{2} \epsilon_{i j}+\frac{\Delta t}{2} \nabla_{h} \cdot\left(\frac{\nabla_{h} w_{i j}}{1+\left|\nabla_{h} w_{i j}\right|^{2}}-\frac{\nabla_{h} z_{i j}}{1+\left|\nabla_{h} z_{i j}\right|^{2}}\right)=0 \\
& 1 \leq i \leq M_{1}, 1 \leq j \leq M_{2} .
\end{aligned}
$$

Taking the inner product of (3.16) with $\epsilon$, we have

$$
\|\epsilon\|^{2}+\frac{\Delta t}{2} \delta\left\|\Delta_{h} \epsilon\right\|^{2}-\frac{\Delta t}{2}\left(\frac{\nabla_{h} w}{1+\left|\nabla_{h} w\right|^{2}}-\frac{\nabla_{h} z}{1+\left|\nabla_{h} z\right|^{2}}, \nabla_{h} \epsilon\right)=0 .
$$

Applying Lemma 3.4, we obtain

$$
\begin{aligned}
\frac{\nabla_{h} w}{1+\left|\nabla_{h} w\right|^{2}}-\frac{\nabla_{h} z}{1+\left|\nabla_{h} z\right|^{2}} & \frac{\nabla_{h} w-\nabla_{h} z+\left|\nabla_{h} z\right|^{2} \nabla_{h} w-\left|\nabla_{h} w\right|^{2} \nabla_{h} z}{\left(1+\left|\nabla_{h} w\right|^{2}\right)\left(1+\left|\nabla_{h} z\right|^{2}\right)} \\
= & \frac{\nabla_{h}(w-z)+\frac{1}{2}\left(\left|\nabla_{h} z\right|^{2}+\left|\nabla_{h} w\right|^{2}\right) \nabla_{h}(w-z)}{\left(1+\left|\nabla_{h} w\right|^{2}\right)\left(1+\left|\nabla_{h} z\right|^{2}\right)} \\
= & \frac{-\frac{1}{2}\left[\left(\nabla_{h} w+\nabla_{h} z\right) \cdot \nabla_{h}(w-z)\right]\left(\nabla_{h} w+\nabla_{h} z\right)}{\left(1+\left|\nabla_{h} w\right|^{2}\right)\left(1+\left|\nabla_{h} z\right|^{2}\right)} .
\end{aligned}
$$

Consequently,

$$
\begin{aligned}
& \left|\frac{\nabla_{h} w}{1+\left|\nabla_{h} w\right|^{2}}-\frac{\nabla_{h} z}{1+\left|\nabla_{h} z\right|^{2}}\right| \\
& \leq \frac{\left|\nabla_{h}(w-z)\right|}{\left(1+\left|\nabla_{h} w\right|^{2}\right)\left(1+\left|\nabla_{h} z\right|^{2}\right)} \\
& \quad \cdot\left[1+\frac{1}{2}\left(\left|\nabla_{h} z\right|^{2}+\left|\nabla_{h} w\right|^{2}\right)+\frac{1}{2}\left(\left|\nabla_{h} w\right|+\left|\nabla_{h} z\right|\right)^{2}\right] \\
& \leq \frac{3}{2}\left|\nabla_{h}(w-z)\right| .
\end{aligned}
$$


It follows from (3.17) and (3.18) that

$$
\begin{aligned}
\|\epsilon\|^{2} & +\frac{\Delta t}{2} \delta\left\|\Delta_{h} \epsilon\right\|^{2} \\
& =\frac{\Delta t}{2}\left(\frac{\nabla_{h} w}{1+\left|\nabla_{h} w\right|^{2}}-\frac{\nabla_{h} z}{1+\left|\nabla_{h} z\right|^{2}}, \nabla_{h} \epsilon\right) \\
& \leq \frac{3 \Delta t}{4}\left\|\Delta_{h} \epsilon\right\| \cdot\|\epsilon\| \\
& \leq \frac{3 \Delta t}{4}\left(\frac{2 \delta}{3}\left\|\Delta_{h} \epsilon\right\|^{2}+\frac{3}{8 \delta}\|\epsilon\|^{2}\right)
\end{aligned}
$$

or

$$
\|\epsilon\|^{2} \leq \frac{9 \Delta t}{32 \delta}\|\epsilon\|^{2} .
$$

When $\Delta t<32 \delta / 9$, it follows that $\|\epsilon\|=0$. Consequently, $\epsilon_{i j}=0,1 \leq i \leq M_{1}$, $1 \leq j \leq M_{2}$. This completes the proof.

Remark 3.6. The constraint on $\Delta t$ in the above theorem may become severe as $\delta$ becomes very small. Meanwhile, there are some related works on MBE models (see e.g., [2, 22, 25]), so that the unique solvability is unconditional in terms of time step. In Section 4, a linearized scheme based on the nonlinear scheme (3.6)-(3.7) will be developed, where the unique solvability is also unconditionally guaranteed.

\subsection{The convergence.}

Lemma 3.7 (Gronwall lemma [23]). Let $\mu$ be positive and let $\nu, a_{k},(k=0,1,2,3, \cdots)$ be nonnegative and satisfy

$$
a_{k} \leq(1+\mu \tau) a_{k-1}+\nu \tau, \quad k=1,2,3, \cdots,
$$

then

$$
a_{k} \leq \exp (\mu k \tau)\left(a_{0}+\nu / \mu\right), \quad k=1,2,3, \cdots .
$$

Define error grid function where $U(x, y, t)$ is the exact solution.

Theorem 3.8. Suppose $U_{i j}^{n}$ is the solution of (1.1)-(1.2) and $u_{i j}^{n}$ is the solution of (3.6) - (3.7). Let

$$
e_{i j}^{n}=U_{i j}^{n}-u_{i, j}^{n}, \quad 1 \leq i \leq M_{1}, \quad 1 \leq j \leq M_{2} .
$$

Then there exists a constant $C_{1}$ independent of $h_{1}, h_{2}$ and $\Delta t$ such as

$$
\left\|e^{n}\right\| \leq C_{1}\left(h_{1}^{2}+h_{2}^{2}++\Delta t^{2}\right), \quad 1 \leq n \leq N .
$$

Proof. Subtracting (3.6), (3.7) from (3.1) and (3.5), respectively, we obtain the error equations

$$
\begin{aligned}
\frac{e_{i j}^{n+1}-e_{i j}^{n}}{\Delta t}+\delta \Delta_{h}^{2} e_{i j}^{n+\frac{1}{2}}+\nabla_{h} \cdot\left[\frac{\nabla_{h} U_{i j}^{n+\frac{1}{2}}}{1+\left|U_{i j}^{n+\frac{1}{2}}\right|^{2}}-\frac{\nabla_{h} u_{i j}^{n+\frac{1}{2}}}{1+\left|u_{i j}^{n+\frac{1}{2}}\right|^{2}}\right]=R_{i j}^{n+\frac{1}{2}}, \\
1 \leq i \leq M_{1}, 1 \leq j \leq M_{2}, 0 \leq n \leq N-1, \\
e_{i j}^{0}=0,1 \leq i \leq M_{1}, 1 \leq j \leq M_{2} .
\end{aligned}
$$


Making a product of (3.20) with $e^{n+\frac{1}{2}}$ and using the summation by parts, we get

$$
\begin{aligned}
& \frac{1}{2 \Delta t}\left(\left\|e^{n+1}\right\|^{2}-\left\|e^{n}\right\|^{2}\right)+\delta\left\|\Delta_{h} e^{n+\frac{1}{2}}\right\|^{2} \\
& \quad=\left(\frac{\nabla_{h} u_{i j}^{n+\frac{1}{2}}}{1+\left|\nabla_{h} u^{n+\frac{1}{2}}\right|^{2}}-\frac{\nabla_{h} U_{i j}^{n+\frac{1}{2}}}{1+\left|\nabla_{h} U^{n+\frac{1}{2}}\right|^{2}}, \nabla_{h} e^{n+\frac{1}{2}}\right)+\left(R^{n+\frac{1}{2}}, e^{n+\frac{1}{2}}\right) .
\end{aligned}
$$

Similarly to the proof of (3.18), we can get

$$
\left(\frac{\nabla_{h} u_{i j}^{n+\frac{1}{2}}}{1+\left|\nabla_{h} u^{n+\frac{1}{2}}\right|^{2}}-\frac{\nabla_{h} U_{i j}^{n+\frac{1}{2}}}{1+\left|\nabla_{h} U^{n+\frac{1}{2}}\right|^{2}}, \nabla_{h} e^{n+\frac{1}{2}}\right) \leq \frac{3}{2}\left\|\nabla_{h} e^{n+\frac{1}{2}}\right\|^{2} .
$$

Inserting (3.23) into (3.22) and using (3.3), we have

$$
\begin{aligned}
\frac{1}{2 \Delta t} & \left(\left\|e^{n+1}\right\|^{2}-\left\|e^{n}\right\|^{2}\right)+\delta\left\|\Delta_{h} e^{n+\frac{1}{2}}\right\|^{2} \\
& =\left(\frac{\nabla_{h} u_{i j}^{n+\frac{1}{2}}}{1+\left|\nabla_{h} u^{n+\frac{1}{2}}\right|^{2}}-\frac{\nabla_{h} U_{i j}^{n+\frac{1}{2}}}{1+\left|\nabla_{h} U^{n+\frac{1}{2}}\right|^{2}}, \nabla_{h} e^{n+\frac{1}{2}}\right)+\left(R^{n+\frac{1}{2}}, e^{n+\frac{1}{2}}\right) \\
& \leq \frac{3}{2}\left\|\nabla_{h} e^{n+\frac{1}{2}}\right\|^{2}+\frac{1}{2}\left\|e^{n+\frac{1}{2}}\right\|^{2}+\frac{1}{2}\left\|R^{n+\frac{1}{2}}\right\|^{2} \\
& \leq \frac{3}{2}\left\|\Delta_{h} e^{n+\frac{1}{2}}\right\| \cdot\left\|e^{n+\frac{1}{2}}\right\|+\frac{1}{2}\left\|e^{n+\frac{1}{2}}\right\|^{2}+\frac{1}{2}|\Omega|\left[c_{1}\left(h_{1}^{2}+h_{2}^{2}+\Delta t^{2}\right)\right]^{2} \\
& \leq \frac{3}{2}\left[\frac{2 \delta}{3}\left\|\Delta_{h} e^{n+\frac{1}{2}}\right\|^{2}+\frac{3}{8 \delta}\left\|e^{n+\frac{1}{2}}\right\|^{2}\right]+\frac{1}{2}\left\|e^{n+\frac{1}{2}}\right\|^{2}+\frac{1}{2}|\Omega|\left[c_{1}\left(h_{1}^{2}+h_{2}^{2}+\Delta t^{2}\right)\right]^{2}
\end{aligned}
$$

or

$$
\frac{1}{2 \Delta t}\left(\left\|e^{n+1}\right\|^{2}-\left\|e^{n}\right\|^{2}\right) \leq\left(\frac{9}{16 \delta}+\frac{1}{2}\right)\left\|e^{n+\frac{1}{2}}\right\|^{2}+\frac{1}{2}|\Omega|\left[c_{1}\left(h_{1}^{2}+h_{2}^{2}+\Delta t^{2}\right)\right]^{2} .
$$

It follows that for $0 \leq n \leq N-1$,

$$
\left[1-\left(\frac{9}{16 \delta}+\frac{1}{2}\right) \Delta t\right]\left\|e^{n+1}\right\|^{2} \leq\left[1+\left(\frac{9}{16 \delta}+\frac{1}{2}\right) \Delta t\right]\left\|e^{n}\right\|^{2}+|\Omega|\left[c_{1}\left(h_{1}^{2}+h_{2}^{2}+\Delta t^{2}\right)\right]^{2} \text {. }
$$

When $\left(\frac{9}{16 \delta}+\frac{1}{2}\right) \Delta t \leq \frac{1}{3}$, it holds that

$$
\left\|e^{n+1}\right\|^{2} \leq\left[1+3\left(\frac{9}{16 \delta}+\frac{1}{2}\right) \Delta t\right]\left\|e^{n}\right\|^{2}+\frac{3}{2}|\Omega|\left[c_{1}\left(h_{1}^{2}+h_{2}^{2}+\Delta t^{2}\right)\right]^{2}, \quad 0 \leq n \leq N-1 .
$$

By Gronwall's lemma, it yields

$$
\left\|e^{n+1}\right\|^{2} \leq e^{3\left(\frac{9}{16 \delta}+\frac{1}{2}\right) T} \cdot \frac{|\Omega| c_{1}^{2}}{\frac{9}{8 \delta}+1}\left(h_{1}^{2}+h_{2}^{2}+\Delta t^{2}\right)^{2}, \quad 0 \leq n \leq N-1
$$

or, equivalently,

$$
\left\|e^{n+1}\right\| \leq e^{\frac{3}{4}\left(\frac{9}{8 \delta}+1\right) T} \cdot \sqrt{\frac{|\Omega|}{\frac{9}{8 \delta}+1}} c_{1}\left(h_{1}^{2}+h_{2}^{2}+\Delta t^{2}\right), \quad 0 \leq n \leq N-1 .
$$

The proof of the theorem is completed. 


\section{LiNEARIZED FOUR-LEVEL SCHEME}

It follows from (1.1)-(1.2) that

$$
u_{t}(x, y, 0)=\delta \Delta^{2} u_{0}(x, y)-\nabla \cdot\left(\frac{\nabla u_{0}(x, y)}{1+\left|\nabla u_{0}(x, y)\right|^{2}}\right) .
$$

Let

$$
\begin{array}{r}
\tilde{u}_{i j}^{\frac{1}{2}}=u_{0}\left(x_{i}, y_{j}\right)+\frac{1}{2} \Delta t u_{t}\left(x_{i}, y_{j}, 0\right), \quad \tilde{u}_{i j}^{\frac{3}{2}}=u_{0}\left(x_{i}, y_{j}\right)+\frac{3}{2} \Delta t u_{t}\left(x_{i}, y_{j}, 0\right), \\
1 \leq i \leq M_{1}, 1 \leq j \leq M_{2} .
\end{array}
$$

Then according to (1.1) and (2.2) with the Taylor expansion, we have

$$
\begin{array}{r}
\frac{U_{i j}^{n+1}-U_{i j}^{n}}{\Delta t}+\delta \Delta_{h}^{2} U_{i j}^{n+\frac{1}{2}}+\nabla_{h} \cdot\left(\frac{\nabla_{h} \tilde{u}_{i j}^{n+\frac{1}{2}}}{1+\left|\nabla_{h} \tilde{u}_{i j}^{n+\frac{1}{2}}\right|^{2}}\right)=\check{R}_{i j}^{n}, \\
1 \leq i \leq M_{1}, 1 \leq j \leq M_{2}, n=0,1, \\
\frac{U_{i j}^{n+1}-U_{i j}^{n}}{\Delta t}+\delta \Delta_{h}^{2} U_{i j}^{n+\frac{1}{2}}+\nabla_{h} \cdot\left(\frac{\nabla_{h} \hat{U}_{i j}^{n+\frac{1}{2}}}{1+\left|\nabla_{h} \hat{U}_{i j}^{n+\frac{1}{2}}\right|^{2}}\right)=\check{R}_{i j}^{n}, \\
1 \leq i \leq M_{1}, 1 \leq j \leq M_{2}, 2 \leq n \leq N-1,
\end{array}
$$

and

$$
\begin{gathered}
\frac{V_{i j}^{n+1}-V_{i j}^{n}}{\Delta t}=\frac{2 \nabla_{h} \tilde{u}_{i j}^{n+\frac{1}{2}} \cdot \nabla_{h} \frac{U_{i j}^{n+1}-U_{i j}^{n}}{\Delta t}}{1+\left|\nabla_{h} \tilde{u}_{i j}^{n+\frac{1}{2}}\right|^{2}} \\
\quad+\check{S}_{i j}^{n}, \quad 1 \leq i \leq M_{1}, 1 \leq j \leq M_{2}, n=0,1, \\
\frac{V_{i j}^{n+1}-V_{i j}^{n}}{\Delta t}=\frac{2 \nabla_{h} \hat{U}_{i j}^{n+\frac{1}{2}} \cdot \nabla_{h} \frac{U_{i j}^{n+1}-U_{i j}^{n}}{\Delta t}}{1+\left|\nabla_{h} \hat{U}_{i j}^{n+\frac{1}{2}}\right|^{2}} \\
+\check{S}_{i j}^{n}, 1 \leq i \leq M_{1}, 1 \leq j \leq M_{2}, 2 \leq n \leq N-1,
\end{gathered}
$$

where there exists a constant $c_{2}$ such that

$$
\begin{array}{cc}
\left|\check{R}_{i j}^{n}\right| \leq c_{2}\left(h_{1}^{2}+h_{2}^{2}+\Delta t^{2}\right), \quad 1 \leq i \leq M_{1}, \quad 1 \leq j \leq M_{2}, \\
\left|\check{S}_{i j}^{n}\right| \leq c_{2}\left(h_{1}^{2}+h_{2}^{2}+\Delta t^{2}\right), \quad 1 \leq i \leq M_{1}, \quad 1 \leq j \leq M_{2} .
\end{array}
$$

Omitting the small terms in (4.1)-(4.4), and noticing the initial conditions

$$
U_{i j}^{0}=u_{0}\left(x_{i}, y_{j}\right), \quad V_{i j}^{0}=\ln \left(1+\left|\nabla_{h} U_{i j}^{0}\right|^{2}\right), \quad 1 \leq i \leq M_{1}, \quad 1 \leq j \leq M_{2},
$$

we construct the difference scheme for (1.1)-(1.2) as follows:

$$
\begin{array}{r}
\frac{u_{i j}^{n+1}-u_{i j}^{n}}{\Delta t}+\delta \Delta_{h}^{2} u_{i j}^{n+\frac{1}{2}}+\nabla_{h} \cdot\left(\frac{\nabla_{h} \tilde{u}_{i j}^{n+\frac{1}{2}}}{1+\left|\nabla_{h} \tilde{u}_{i j}^{n+\frac{1}{2}}\right|^{2}}\right)=0, \\
1 \leq i \leq M_{1}, 1 \leq j \leq M_{2}, n=0,1,
\end{array}
$$




$$
\begin{gathered}
\frac{u_{i j}^{n+1}-u_{i j}^{n}}{\Delta t}+\delta \Delta_{h}^{2} u_{i j}^{n+\frac{1}{2}}+\nabla_{h} \cdot\left(\frac{\nabla_{h} \hat{u}_{i j}^{n+\frac{1}{2}}}{1+\left|\nabla_{h} \hat{u}_{i j}^{n+\frac{1}{2}}\right|^{2}}\right)=0, \\
1 \leq i \leq M_{1}, 1 \leq j \leq M_{2}, 2 \leq n \leq N-1, \\
u_{i j}^{0}=u_{0}\left(x_{i}, y_{j}\right), \quad 1 \leq i \leq M_{1}, \quad 1 \leq j \leq M_{2},
\end{gathered}
$$

and

$$
\begin{gathered}
\frac{v_{i j}^{n+1}-v_{i j}^{n}}{\Delta t}=\frac{2 \nabla_{h} \tilde{u}_{i j}^{n+\frac{1}{2}} \cdot \nabla_{h} \frac{u_{i j}^{n+1}-u_{i j}^{n}}{\Delta t}}{1+\left|\nabla_{h} \tilde{u}_{i j}^{n+\frac{1}{2}}\right|^{2}}, \\
1 \leq i \leq M_{1}, 1 \leq j \leq M_{2}, n=0,1, \\
v_{i j}^{0}=\ln \left(1+\left|\nabla_{h} u_{i j}^{0}\right|^{2}\right), 1 \leq i \leq M_{1}, 1 \leq j \leq M_{2}, \\
\frac{v_{i j}^{n+1}-v_{i j}^{n}}{\Delta t}=\frac{2 \nabla_{h} \hat{u}_{i j}^{n+\frac{1}{2}} \cdot \nabla_{h} \frac{u_{i j}^{n+1}-u_{i j}^{n}}{\Delta t}}{1+\left|\nabla_{h} \hat{u}_{i j}^{n+\frac{1}{2}}\right|^{2}}, \\
1 \leq i \leq M_{1}, 1 \leq j \leq M_{2}, 2 \leq n \leq N-1 .
\end{gathered}
$$

Remark 4.1. Our proposed schemes 3.6)-(3.7) and (4.8)-(4.9) do not use a compact stencil for the calculation of the approximation of the second-order term $\nabla \cdot\left(\frac{\nabla u}{1+|\nabla u|^{2}}\right)$. In $[22$, a compact stencil is used by introducing an auxiliary variable $w=\Delta u$. This approach will further improve the stability of numerical schemes. In our numerical schemes, we discretize the fourth-order term directly instead of using the auxiliary variable $w=\Delta u$, which will sample points from $(i-2, j),(i-1, j),(i, j),(i+1, j),(i+2, j)$, in addition to other lattice points in the neighborhood of the point $(i, j)$, even if the compact stencil is used for the calculation of the approximation of the second-order term $\nabla \cdot\left(\frac{\nabla u}{1+|\nabla u|^{2}}\right)$, and in the resulting linear system, the discretization of the fourth-order term will dominate comparing to the discretization of the second-order terms. So without including the auxiliary variable $w=\Delta u$, the long stencil approximation and compact stencil approximation will not give much difference on numerical solutions. We will consider the approach with auxiliary variable $w=\Delta u$ and the compact stencil presented in [22] in our future study of this type of PDEs.

\subsection{The stability and solvability.}

Theorem 4.2. The solution of the difference scheme (4.8)-(4.12) satisfies the energy law (2.5) in the discrete sense,

$$
\left\|\frac{u^{n+1}-u^{n}}{\Delta t}\right\|^{2}+\frac{E_{h}^{n+1}-E_{h}^{n}}{\Delta t}=0,
$$

where $E_{h}^{n}$ is defined in Theorem 3.1 .

Proof. It is similar to the proof of Theorem 3.1

Theorem 4.3. The linearized scheme (4.8)-(4.10) is uniquely solvable. 
Proof. Suppose $u^{n}$ has been determined. Then (4.8)-4.9) is a linear system about $u^{n+1}$. Consider its homogenous system

$$
\frac{u_{i j}^{n+1}}{\Delta t}+\frac{\delta}{2} \Delta_{h}^{2} u_{i j}^{n+1}=0, \quad 1 \leq i \leq M_{1}, 1 \leq j \leq M_{2} .
$$

Making inner product with $u^{n+1}$, we get

$$
\frac{1}{\Delta t}\left\|u^{n+1}\right\|^{2}+\frac{\delta}{2}\left\|\Delta_{h} u^{n+1}\right\|^{2}=0 .
$$

Thus $u^{n+1}=0$. This completes the proof.

4.2. The convergence. For the convergence of the difference scheme, we have the following result.

Theorem 4.4. The difference scheme (4.8)-(4.10) is convergent and there exists a constant $C_{2}$ independent of the step sizes such that

$$
\left\|e^{n}\right\| \leq C_{2}\left(h_{1}^{2}+h_{2}^{2}+\Delta t^{2}\right), \quad 1 \leq n \leq N
$$

Proof. Let

$$
e_{i j}^{n}=U_{i j}^{n}-u_{i j}^{n}, \quad 1 \leq i \leq M_{1}, \quad 1 \leq j \leq M_{2}, \quad 0 \leq n \leq N .
$$

Subtracting (4.8), (4.9) and (4.10) from (4.1), (4.2) and (4.7), respectively, we obtain the error equations

$$
\begin{gathered}
\frac{e_{i j}^{n+1}-e_{i j}^{n}}{\Delta t}+\delta \Delta_{h}^{2} e_{i j}^{n+\frac{1}{2}}=\check{R}_{i j}^{n}, 1 \leq i \leq M_{1}, 1 \leq j \leq M_{2}, n=0,1, \\
\frac{e_{i j}^{n+1}-e_{i j}^{n}}{\Delta t}+\delta \Delta_{h}^{2} e_{i j}^{n+\frac{1}{2}} \\
+\nabla_{h} \cdot\left(\frac{\nabla_{h} \hat{U}_{i j}^{n+\frac{1}{2}}}{1+\left|\nabla_{h} \hat{U}_{i j}^{n+\frac{1}{2}}\right|^{2}}-\frac{\nabla_{h} \hat{u}_{i j}^{n+\frac{1}{2}}}{1+\left|\nabla_{h} \hat{u}_{i j}^{n+\frac{1}{2}}\right|^{2}}\right)=\check{R}_{i j}^{n}, \\
1 \leq i \leq M_{1}, 1 \leq j \leq M_{2}, 2 \leq n \leq N-1, \\
e_{i j}^{0}=0, \quad 1 \leq i \leq M_{1}, \quad 1 \leq j \leq M_{2} .
\end{gathered}
$$

Taking the inner product of (4.15) with $e^{n+\frac{1}{2}}$, we have

$$
\frac{1}{2 \Delta t}\left(\left\|e^{n+1}\right\|^{2}-\left\|e^{n}\right\|^{2}\right)+\delta\left\|\Delta_{h} e^{n+\frac{1}{2}}\right\|^{2}=\left(\check{R}^{n+\frac{1}{2}}, e^{n+\frac{1}{2}}\right), \quad n=0,1 .
$$

Taking the inner product of (4.16) with $e^{n+\frac{1}{2}}$, we have

$$
\begin{aligned}
& \frac{1}{2 \Delta t}\left(\left\|e^{n+1}\right\|^{2}-\left\|e^{n}\right\|^{2}\right)+\delta\left\|\Delta_{h} e^{n+\frac{1}{2}}\right\|^{2} \\
&=\left(\frac{\nabla_{h} \hat{U}^{n+\frac{1}{2}}}{1+\left|\nabla_{h} \hat{U}^{n+\frac{1}{2}}\right|^{2}}-\frac{\nabla_{h} \hat{u}^{n+\frac{1}{2}}}{1+\left|\nabla_{h} \hat{u}^{n+\frac{1}{2}}\right|^{2}}, \nabla_{h} e^{n+\frac{1}{2}}\right)+\left(\check{R}^{n+\frac{1}{2}}, e^{n+\frac{1}{2}}\right), \\
& 2 \leq n \leq N-1 .
\end{aligned}
$$


Similarly to the proof of (3.18), we can get

$$
\begin{aligned}
& \left(\frac{\nabla_{h} \hat{U}^{n+\frac{1}{2}}}{1+\left|\nabla_{h} \hat{U}^{n+\frac{1}{2}}\right|^{2}}-\frac{\nabla_{h} \hat{u}^{n+\frac{1}{2}}}{1+\left|\nabla_{h} \hat{u}^{n+\frac{1}{2}}\right|^{2}}, \nabla_{h} e^{n+\frac{1}{2}}\right) \\
& \quad \leq \frac{3}{2}\left\|\nabla_{h} \hat{e}^{n+\frac{1}{2}}\right\| \cdot\left\|\nabla_{h} e^{n+\frac{1}{2}}\right\|, \quad 2 \leq n \leq N-1 .
\end{aligned}
$$

Inserting (4.20) into (4.19), we have

$$
\begin{aligned}
\frac{1}{2 \Delta t}( & \left.\left\|e^{n+1}\right\|^{2}-\left\|e^{n}\right\|^{2}\right)+\delta\left\|\Delta_{h} e^{n+\frac{1}{2}}\right\|^{2} \\
\leq & \frac{3}{2}\left\|\nabla_{h} \hat{e}^{n+\frac{1}{2}}\right\| \cdot\left\|\nabla_{h} e^{n+\frac{1}{2}}\right\|+\left(\check{R}^{n+\frac{1}{2}}, e^{n+\frac{1}{2}}\right) \\
\leq & \frac{3}{4}\left(\left\|\nabla_{h} \hat{e}^{n+\frac{1}{2}}\right\|^{2}+\left\|\nabla_{h} e^{n+\frac{1}{2}}\right\|^{2}\right)+\left(\check{R}^{n+\frac{1}{2}}, e^{n+\frac{1}{2}}\right) \\
\leq & \frac{3}{4}\left(\left\|\Delta_{h} \hat{e}^{n+\frac{1}{2}}\right\| \cdot\left\|\hat{e}^{n+\frac{1}{2}}\right\|+\left\|\Delta_{h} e^{n+\frac{1}{2}}\right\| \cdot\left\|e^{n+\frac{1}{2}}\right\|\right)+\left(\check{R}^{n+\frac{1}{2}}, e^{n+\frac{1}{2}}\right) \\
\leq & \frac{3}{4}\left(\frac{\delta}{15}\left\|\Delta_{h} \hat{e}^{n+\frac{1}{2}}\right\|^{2}+\frac{15}{4 \delta}\left\|\hat{e}^{n+\frac{1}{2}}\right\|^{2}+\frac{2 \delta}{3}\left\|\Delta_{h} e^{n+\frac{1}{2}}\right\|^{2}+\frac{3}{8 \delta}\left\|e^{n+\frac{1}{2}}\right\|^{2}\right) \\
& +\left(\check{R}^{n+\frac{1}{2}}, e^{n+\frac{1}{2}}\right) \\
= & \frac{\delta}{20}\left\|\Delta_{h} \hat{e}^{n+\frac{1}{2}}\right\|^{2}+\frac{\delta}{2}\left\|\Delta_{h} e^{n+\frac{1}{2}}\right\|^{2}+\frac{45}{16 \delta}\left\|\hat{e}^{n+\frac{1}{2}}\right\|^{2}+\frac{9}{32 \delta}\left\|e^{n+\frac{1}{2}}\right\|^{2} \\
& +\left(\check{R}^{n+\frac{1}{2}}, e^{n+\frac{1}{2}}\right) \\
\leq & \frac{\delta}{20}\left(8\left\|\Delta_{h} e^{n-\frac{1}{2}}\right\|^{2}+2\left\|\Delta_{h} e^{n-\frac{3}{2}}\right\|^{2}\right)+\frac{\delta}{2}\left\|\Delta_{h} e^{n+\frac{1}{2}}\right\|^{2} \\
& +\frac{45}{16 \delta}\left(8\left\|e^{n-\frac{1}{2}}\right\|^{2}+2\left\|e^{n-\frac{3}{2}}\right\|^{2}\right) \\
& +\frac{9}{32 \delta}\left\|e^{n+\frac{1}{2}}\right\|^{2}+\left(\check{R}^{n+\frac{1}{2}}, e^{n+\frac{1}{2}}\right), \quad 2 \leq n \leq N-1 .
\end{aligned}
$$

Summing (4.18) and (4.21), we get

$$
\begin{aligned}
& \frac{1}{2 \Delta t}\left(\left\|e^{n+1}\right\|^{2}-\left\|e^{0}\right\|^{2}\right) \\
& \leq \frac{45}{16 \delta}\left(8 \sum_{l=2}^{n}\left\|e^{l-\frac{1}{2}}\right\|^{2}+2 \sum_{l=2}^{n}\left\|e^{l-\frac{3}{2}}\right\|^{2}\right) \\
&+\frac{9}{32 \delta} \sum_{l=2}^{n}\left\|e^{l+\frac{1}{2}}\right\|^{2}+\sum_{l=0}^{n}\left(\check{R}^{l+\frac{1}{2}}, e^{l+\frac{1}{2}}\right) \\
& \leq \frac{45}{16 \delta}\left(8 \sum_{l=1}^{n-1}\left\|e^{l+\frac{1}{2}}\right\|^{2}+2 \sum_{l=0}^{n-2}\left\|e^{l+\frac{1}{2}}\right\|^{2}\right)+\frac{9}{32 \delta} \sum_{l=2}^{n}\left\|e^{l+\frac{1}{2}}\right\|^{2} \\
&+\frac{1}{2} \sum_{l=0}^{n}\left(\left\|\check{R}^{l+\frac{1}{2}}\right\|^{2}+\left\|e^{l+\frac{1}{2}}\right\|^{2}\right) \\
& \leq \frac{1}{2}\left(\frac{9}{32 \delta}+\frac{1}{2}\right)\left\|e^{n+1}\right\|^{2}+\left(\frac{909}{32 \delta}+\frac{1}{2}\right) \sum_{l=0}^{n}\left\|e^{l}\right\|^{2}+\frac{1}{2} \sum_{l=0}^{n}\left\|\check{R}^{l+\frac{1}{2}}\right\|^{2} .
\end{aligned}
$$


Noticing $\left\|e^{0}\right\|=0$ and (4.5), we have

$$
\begin{aligned}
{\left[1-\left(\frac{9}{32 \delta}+\frac{1}{2}\right) \Delta t\right] } & \left\|e^{n+1}\right\|^{2} \leq\left(\frac{909}{32 \delta}+\frac{1}{2}\right) \Delta t \sum_{l=1}^{n}\left\|e^{l}\right\|^{2} \\
& +(n+1) \Delta t\left[c_{2}\left(h_{1}^{2}+h_{2}^{2}+\Delta t^{2}\right)\right]^{2}, 0 \leq n \leq N-1 .
\end{aligned}
$$

When $\left(\frac{9}{32 \delta}+\frac{1}{2}\right) \Delta t \leq \frac{1}{3}$, we have

$$
\begin{aligned}
\left\|e^{n+1}\right\|^{2} \leq & \frac{3}{2}\left(\frac{909}{32 \delta}+\frac{1}{2}\right) \Delta t \sum_{l=1}^{n}\left\|e^{l}\right\|^{2} \\
& +\frac{3}{2}(n+1) \Delta t\left[c_{2}\left(h_{1}^{2}+h_{2}^{2}+\Delta t^{2}\right)\right]^{2}, \quad 0 \leq n \leq N-1 .
\end{aligned}
$$

The Gronwall lemma yields

$$
\left\|e^{n+1}\right\|^{2} \leq e^{\frac{3}{2}\left(\frac{909}{32 \delta}+\frac{1}{2}\right) T} \cdot \frac{1}{\frac{909}{32 \delta}+\frac{1}{2}} \cdot\left[c_{2}\left(h_{1}^{2}+h_{2}^{2}+\Delta t^{2}\right)\right]^{2}, \quad 0 \leq n \leq N-1
$$

or

$$
\left\|e^{n+1}\right\| \leq e^{\frac{3}{4}\left(\frac{909}{32 \delta}+\frac{1}{2}\right) T} \cdot \frac{1}{\sqrt{\frac{909}{32 \delta}+\frac{1}{2}}} \cdot c_{2}\left(h_{1}^{2}+h_{2}^{2}+\Delta t^{2}\right) \quad 0 \leq n \leq N-1 .
$$

This completes the proof.

\section{NumericAl EXPERIMENTS}

The purpose of this section is to verify the theoretical claims of the proposed numerical schemes. We begin by presenting a numerical test for the problem (1.1)(1.2) in one space dimension, which gives the evidence of the expected energy stability and second-order convergence of the proposed numerical scheme. In a second test, we reproduce some calculations from a previous paper [15. Finally, we perform a coarsening dynamics simulation.

We have carried out the numerical tests with both the nonlinear scheme (3.6)(3.7) and the linear scheme (4.8)-(4.10). Both schemes give similar numerical results. For the nonlinear scheme, we use Newton's iterative solver. The numerical solution at the previous time level is taken as the initial guess at each time step. At each Newton iteration step, an algebraic multigrid solver (AMG) is employed to solve the resulted linear system. The tolerance of the Newton iteration is set to be $10^{-6}$, and the tolerance of the AMG solver is $10^{-8}$. This Newton iteration method with the AMG solver has been successfully used for many simulations in our previous work; see e.g., [18,19. In this section, we will only show the numerical results obtained by the linear scheme (4.8)-(4.10).

Example 5.1. Consider the one-dimensional MBE model without slope selection:

$$
\begin{array}{ll}
\frac{\partial u}{\partial t}=-\left(\frac{u_{x}}{1+\left|u_{x}\right|^{2}}\right)_{x}-\delta u_{x x x x}, & (x, t) \in(0,12) \times(0, T), \\
u(x, 0)=0.1\left(\sin \frac{\pi x}{2}+\sin \frac{2 \pi x}{3}+\sin \pi x\right), & x \in[0,12]
\end{array}
$$

subject to periodic boundary conditions, where $\delta=0.1$. 
TABLE 1. Example 5.1; numerical accuracy test in time

\begin{tabular}{|c|c|c|}
\hline$\Delta t$ & $L_{2}$-error & order \\
\hline 0.01 & $1.2320 \mathrm{E}-3$ & - \\
\hline 0.005 & $2.6977 \mathrm{E}-4$ & 2.1912 \\
\hline 0.0025 & $6.3541 \mathrm{E}-5$ & 2.0860 \\
\hline 0.00125 & $1.5416 \mathrm{E}-5$ & 2.0431 \\
\hline
\end{tabular}

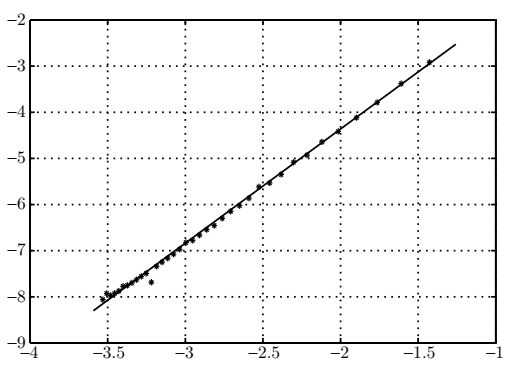

Figure 1. Example 5.1: The log-log plot of the error in $L_{2}$-norm versus the mesh size $12 / M_{1}$. The solid line is the linear leastsquares fitting whose slope is the average order of accuracy in space, which is 2.0840 .

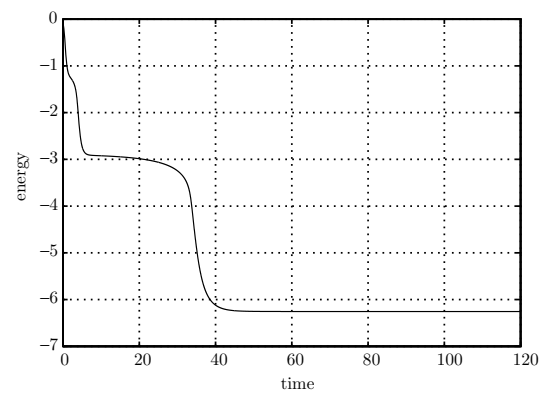

Figure 2. Example 5.1 energy curve, $0 \leq t \leq 120$.

To test the accuracy we take the numerical solution obtained by using $M_{1}=1200$ and $\Delta t=10^{-5}$ as the reference solution. In Table 1, we show the grid refinement analysis in time of our numerical scheme with $M_{1}=1200$. We can see that the method is second-order accurate in time in a discrete $L_{2}$-norm.

To show the convergence order in space, we use a linear least-squares fitting to find the average order of accuracy:

$$
\log \| \text { error } \|_{2} \approx \text { order } \log \left(12 / M_{1}\right)+C
$$

in Figure 1] We choose $M_{1}=50+10 k, k=0,1,2, \ldots, 36$. The order is found to be 2.0840 and the constant is $C=-0.7003$.

The discrete energy is presented in Figure 2 with $\Delta t=0.01$ and $M_{1}=120$. It is monotonically nonincreasing in time, which demonstrates the stability analysis. 

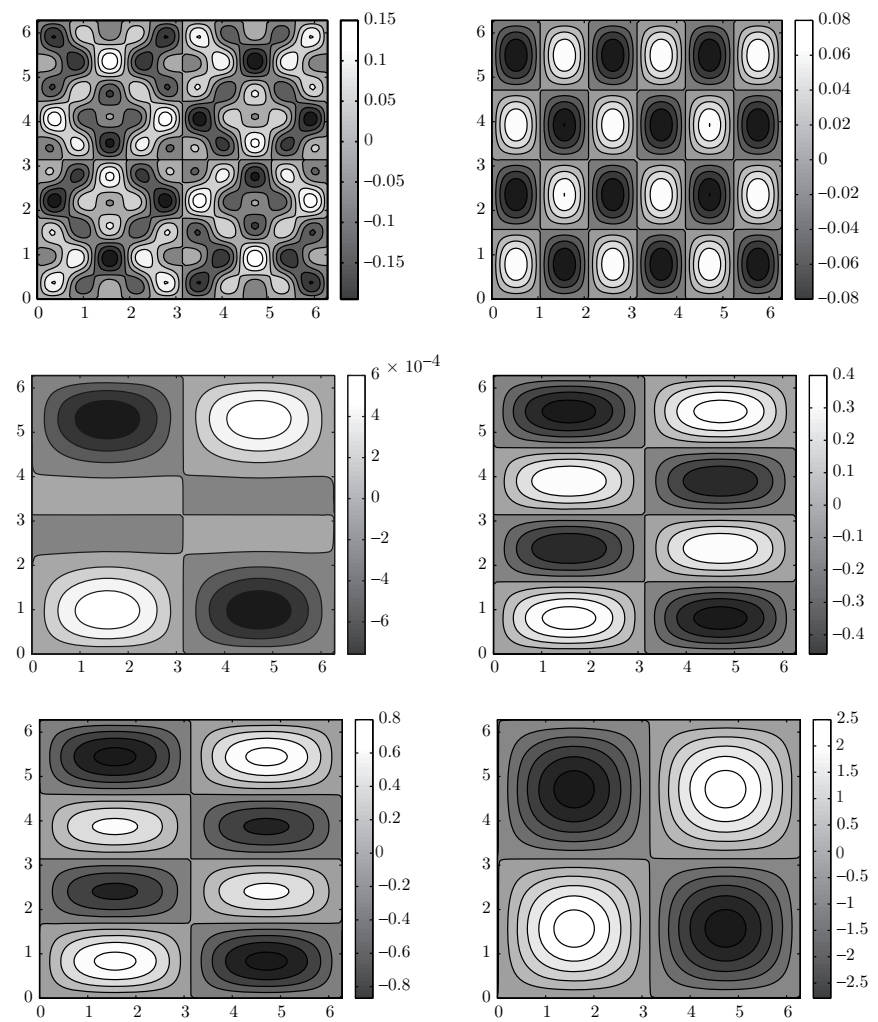

Figure 3. Example 5.2 contour plots of the numerical solution at $t=0,0.05,2.5,5.5,8$ and 30 .

Example 5.2. Consider the two-dimensional MBE model without slope selection. Here $\delta=0.1$ and the domain is $\Omega=[0,2 \pi] \times[0,2 \pi]$. The initial condition is

$$
u_{0}(x, y)=0.1(\sin 3 x \sin 2 x+\sin 5 x \sin 5 y) \quad(x, y) \in \Omega .
$$

This example has been studied by Li and Liu [15] for observing the nonlinear interaction. We show contour plots of the numerical solution $u$ and the magnitude of its gradient $|\nabla u|$ at $t=0,0.05,2.5,5.5,8$ and 30 with $\Delta t=0.01$ and $M_{1}=M_{2}=$ 100 in Figure 3 and Figure 4, respectively. The structural transition of mounds in the coarsening can be observed in Figure 3. In Figure 4, it can be seen that the magnitude of the gradient $|\nabla u|$ is much larger than that for the thin film epitaxy growth with slope selection; see [15].

The energy evolution is shown in Figure 5. We observe that the energy decays rapidly initially. Then after a relatively long period of time, the energy has its second sharp decay at around $t=5$. After the third fast decay, the energy reaches a steady state after $t=15$.

The contour plots figures in Figure 3 and Figure 4 and the energy evolution profile shown in Figure 5, obtained by our proposed numerical scheme, are in good agreement with those in 15 . 

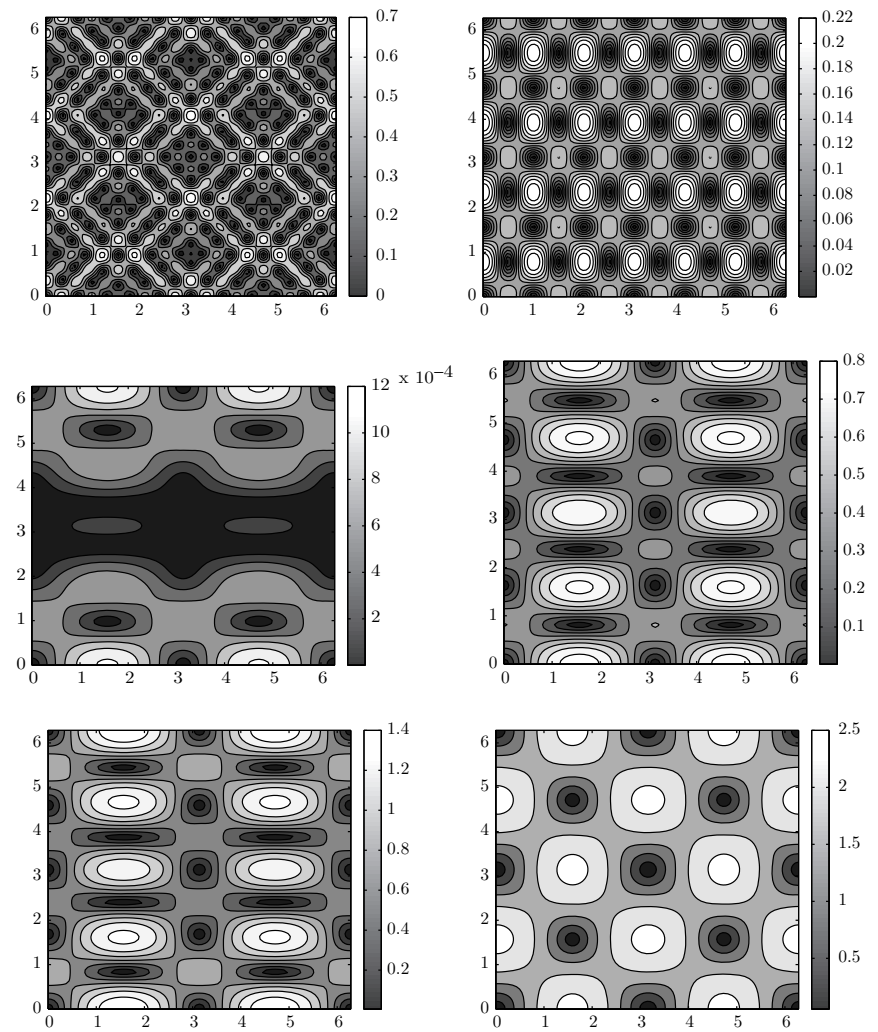

Figure 4. Example 5.2 contour plots of gradient at $t=0$, $0.05,2.5,5.5,8$ and 30 .
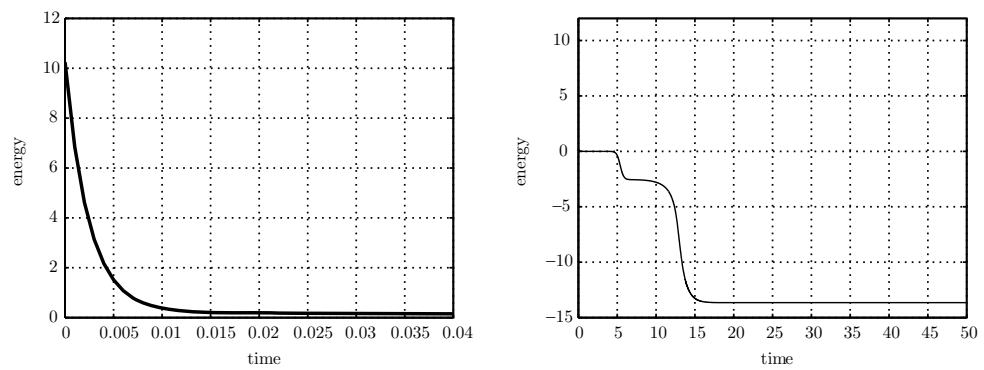

Figure 5. Example 5.2 energy evolution. Left: energy decays in early stage $0 \leq t \leq 0.04$. Right: energy decays for $0 \leq t \leq 50$.

Example 5.3. (coarsening dynamics). We simulate the no-slope-selection MBE model (1.1) in two dimensions. The initial condition is a random state by random numbers varying from -0.001 to 0.001 on each grid point. The domain is $\Omega=$ $[0,12.8] \times[0,12.8]$ and also the problem is subject to periodic boundary conditions. We use a $300 \times 300$ grid in this simulation. For the temporal step size $\Delta t$, we use $\Delta t=0.001$ on the time interval $[0,400], \Delta t=0.01$ on the time interval $[400,6000]$ and $\Delta t=0.1$ for $t>6000$. Figure 6 presents time snapshots of the computed film 
height $u$ with $\delta=0.001$. Coarsening dynamics with shapes of hills and valleys in the system is evident. The system clearly saturates to a one-hill-one-valley structure at $t=100000$.

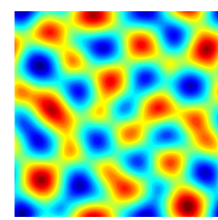

(a) $t=400$

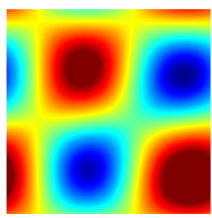

(d) $t=20000$

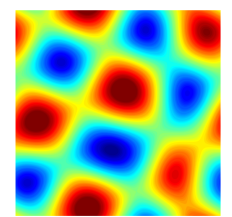

(b) $t=4000$

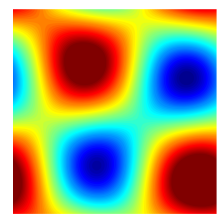

(e) $t=40000$

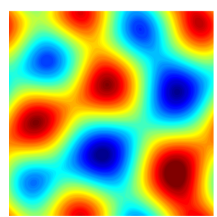

(c) $t=10000$

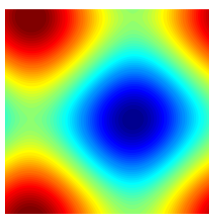

(f) $t=100000$

FiguRE 6. Example 5.3. time snapshots of the computed height function $u$ with the parameters $\Omega=[0,12.8] \times[0,12.8] ; \delta=0.001$.

The energy decay rate, the growth rates of surface roughness and mound width are of great interest to physicists and engineers. The surface roughness is defined as

$$
w(t)=\sqrt{\frac{1}{|\Omega|} \int_{\Omega}|u(\mathbf{x}, t)-\bar{u}(t)|^{2} d \mathbf{x},}, \quad \text { with } \quad \bar{u}(t)=\frac{1}{|\Omega|} \int_{\Omega} u(\mathbf{x}, t) d \mathbf{x},
$$

where $\mathbf{x}=(x, y)^{T}$. The mound width $\lambda(t)$ measures the mean size of the network cell, which can be calculated as suggested in 9,27 from the height-height correlation function

$$
K_{u u}(\mathbf{r}, t)=\int_{\Omega} u(\mathbf{x}+\mathbf{r}, t) u(\mathbf{x}, t) d \mathbf{x},
$$

where $\mathbf{r}$ is a positive vector. In our computation, we take a simple form $\mathbf{r}=(r, r)^{T}$. $K_{u u}$ can be regarded as a function of $\mathbf{r}$ for fixed $t$, which shows an oscillatory character reflecting the presence of a mound structure. For a given $t$, the mean pyramid width $\lambda(t)$ is defined as $r_{0}(t)$, which is the first zero crossing of $K_{u u}(\mathbf{r}, t)$,

$$
r_{0}(t)=\inf \left\{r>0, K_{u u}(\mathbf{r}, t)=0\right\} .
$$

For the no-slope-selection MBE model (1.1), one obtains $w(t) \sim \mathcal{O}\left(t^{1 / 2}\right), \lambda(t) \sim$ $\mathcal{O}\left(t^{1 / 4}\right)$ and the energy (1.3) evolves like $E(t) \sim \mathcal{O}(-\ln t)$ 9, 15, 16. In Figure 7(a), we present the linear least-squares fit of the semi-log energy data up to $t=4000$. The fitted line has the form $m e \ln t+b e$, with $m e=-40.01$, be $=-110.3$. In Figure 7 (b), we present the linear least-squares fit of the log-log surface roughness data up to $t=4000$. The fitted line has the form $b r t^{m r}$, where $b r=0.4089$ and $m r=0.5021$. Figure 7 (c) gives the linear least-squares fit of the log-log width data up to $t=4000$. The fitted line has the form $b w t^{m w}$, where $b w=0.2313$ 
and $m w=0.2412$. A clear explanation of the $-\ln t, t^{1 / 2}$ and $t^{1 / 4}$ scaling laws for the energy decay rate, the surface roughness growth rate and mound width growth rate, respectively, has been supported by our simulation. Figures 7(a) and 7(b) also agree well with those in [2,3].

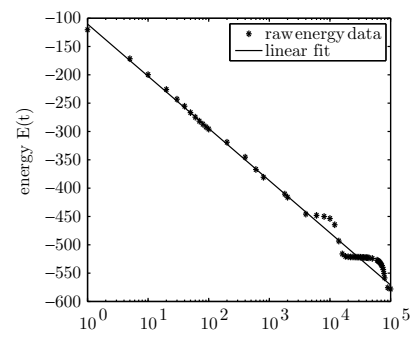

(a)

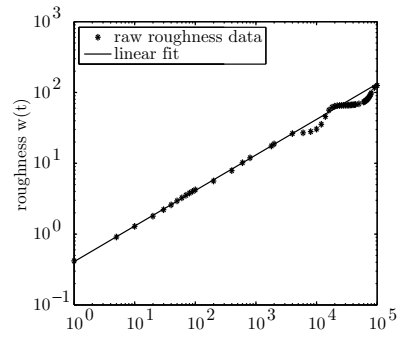

(b)

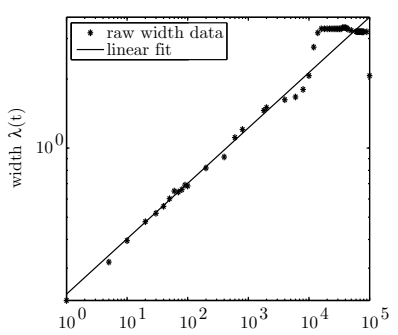

(c)

Figure 7. Example 5.3 energy, roughness and width evolution for the simulation depicted in Figure 6. The final simulation time is $t=100000$.

\section{Conclusion}

In this work, we have developed and analyzed two energy stable schemes for solving molecular beam epitaxy model without slope selection. One scheme is nonlinear, while the other one is fully linear. Both schemes are proved to be uniquely solvable and of second-order convergence in a discrete $L_{2}$-norm. By introducing an auxiliary variable $v$, we define the physical energy in an alternate way, which avoids the classical theoretical difficulty of energy stability. However, since the auxiliary variable $v$ is updated by (3.8), which is based on the evolutionary equation (2.2) instead of the original representation (2.1), the energy stability result is obtained based on a modified energy. Such a modified energy stability is an inferior result, compared to a direct energy stability in terms of the original variable $u$. Several numerical experiments are carried out in Section 5. The numerical results are in good agreement with the existing ones and the theoretical claims have been demonstrated numerically. We also perform a long time simulation for a coarsening process. The $t^{1 / 2}$ growth law for surface roughness, the $t^{1 / 4}$ growth law for mound width and the $-\ln (t)$ decay law for energy of the no-slope-selection MBE model (1.1) have been recovered and verified numerically.

We should point out that the $H^{2}$ numerical stability for the solution $u$ cannot be derived at the theoretical level, while for the original PDE solution (1.1), it is well-known that the energy stability indicates a global in time $H^{2}$ stability for the solution $u$. Meanwhile, there are some related references in existing literatures, of energy stable schemes to (1.1) so that an $H^{2}$ stability analysis is available for the solution $u$, with either first- or second-order accuracy in time; see e.g., [2, 22, 25]. One of the future works in this direction is to develop energy stable schemes, especially high-order linear energy stable schemes, based on a direct energy discretization in terms of the original variable $u$. Other future works in this direction include carrying out the adaptive time integration as that in [19], i.e., treating the fast dynamics 
changes and slow changes separately. This is important in further improving the efficiency for the large time simulations.

\section{ACKNOWLEDGEMENTS}

The authors would like to express their deep thanks to the anonymous reviewers whose valuable comments and suggestions helped them improve this article greatly.

\section{REFERENCES}

[1] Georgios D. Akrivis, Finite difference discretization of the cubic Schrödinger equation, IMA J. Numer. Anal. 13 (1993), no. 1, 115-124, DOI 10.1093/imanum/13.1.115. MR.1199033 (94d:65046)

[2] Wenbin Chen, Sidafa Conde, Cheng Wang, Xiaoming Wang, and Steven M. Wise, A linear energy stable scheme for a thin film model without slope selection, J. Sci. Comput. 52 (2012), no. 3, 546-562, DOI 10.1007/s10915-011-9559-2. MR.2948706

[3] W.B. Chen, C. Wang, X.M. Wang, S.M. Wise, A linear iteration algorithm for a second-order energy stable scheme for a thin film model without slope selection, J. Sci. Comput. 54 (2014), no. 3, 574-601, DOI 10.1007/s10915-013-9774-0. MR3199497

[4] Qiang Du and R. A. Nicolaides, Numerical analysis of a continuum model of phase transition, SIAM J. Numer. Anal. 28 (1991), no. 5, 1310-1322, DOI 10.1137/0728069. MR1119272 (92h:65166)

[5] J.W. Evans and P.A. Thiel, A little chemistry helps the big get Bigger, Science, 330 (2010), 599-600.

[6] J.W. Evans, P.A. Thiel and M.C. Bartelt, Morphological evolution during epitaxial thin film growth: formation of $2 D$ islands and $3 D$ mounds, Surf. Sci. Rep., 61 (2006), 1-128.

[7] David J. Eyre, Unconditionally gradient stable time marching the Cahn-Hilliard equation, Computational and Mathematical Models of Microstructural Evolution (San Francisco, CA, 1998), Mater. Res. Soc. Sympos. Proc., vol. 529, MRS, Warrendale, PA, 1998, pp. 39-46, DOI 10.1557/PROC-529-39. MR1676409

[8] Daisuke Furihata, A stable and conservative finite difference scheme for the Cahn-Hilliard equation, Numer. Math. 87 (2001), no. 4, 675-699, DOI 10.1007/PL00005429. MR1815731 (2001m:65106)

[9] L. Golubović, Interfacial coarsening in epitaxial growth models without slope selection, Phys. Rev. Lett., 78 (1997), 90-93.

[10] F. Guillén-González and G. Tierra, On linear schemes for a Cahn-Hilliard diffuse interface model, J. Comput. Phys. 234 (2013), 140-171, DOI 10.1016/j.jcp.2012.09.020. MR2999772

[11] Yinnian He, Yunxian Liu, and Tao Tang, On large time-stepping methods for the Cahn-Hilliard equation, Appl. Numer. Math. 57 (2007), no. 5-7, 616-628, DOI 10.1016/j.apnum.2006.07.026. MR.2322435 (2008g:65134)

[12] Z. Hu, S. M. Wise, C. Wang, and J. S. Lowengrub, Stable and efficient finite-difference nonlinear-multigrid schemes for the phase field crystal equation, J. Comput. Phys. 228 (2009), no. 15, 5323-5339, DOI 10.1016/j.jcp.2009.04.020. MR2541456 (2010e:80009)

[13] M.D. Johnson, C. Orme, A.W. Hunt, D. Graff, J. Sudijono, L.M. Sander and B.G. Orr, Stable and unstable growth in molecular beam epitaxy, Phys. Rev. Lett., 72 (1994), 116-119.

[14] Robert V. Kohn and Xiaodong Yan, Upper bound on the coarsening rate for an epitaxial growth model, Comm. Pure Appl. Math. 56 (2003), no. 11, 1549-1564, DOI 10.1002/cpa.10103. MR.1995869(2004d:35043)

[15] Bo Li and Jian-Guo Liu, Thin film epitaxy with or without slope selection, European J. Appl. Math. 14 (2003), no. 6, 713-743, DOI 10.1017/S095679250300528X. MR2034852 (2004m:35147)

[16] Bo Li and Jian-Guo Liu, Epitaxial growth without slope selection: energetics, coarsening, and dynamic scaling, J. Nonlinear Sci. 14 (2004), no. 5, 429-451 (2005), DOI 10.1007/s00332004-0634-9. MR2126166 (2005k:35202)

[17] Juan Li, ZhiZhong Sun, and Xuan Zhao, A three level linearized compact difference scheme for the Cahn-Hilliard equation, Sci. China Math. 55 (2012), no. 4, 805-826, DOI 10.1007/s11425011-4290-x. MR 2903464 
[18] Zhonghua Qiao, Zhi-zhong Sun, and Zhengru Zhang, The stability and convergence of two linearized finite difference schemes for the nonlinear epitaxial growth model, Numer. Methods Partial Differential Equations 28 (2012), no. 6, 1893-1915, DOI 10.1002/num.20707. MR2981875

[19] Zhonghua Qiao, Zhengru Zhang, and Tao Tang, An adaptive time-stepping strategy for the molecular beam epitaxy models, SIAM J. Sci. Comput. 33 (2011), no. 3, 1395-1414, DOI 10.1137/100812781. MR2813245 (2012h:65164)

[20] L. Ratke and P. W. Voorhees, Growth and coarsening, Springer-Verlag, Berlin, 2002.

[21] A. A. Samarskiı̌ and V. B. Andreev, Raznostnye Metody dlya Ellipticheskikh Uravnenii (Russian), Izdat. 'Nauka', Moscow, 1976. MR0502017 (58 \#19209a)

[22] Jie Shen, Cheng Wang, Xiaoming Wang, and Steven M. Wise, Second-order convex splitting schemes for gradient flows with Ehrlich-Schwoebel type energy: application to thin film epitaxy, SIAM J. Numer. Anal. 50 (2012), no. 1, 105-125, DOI 10.1137/110822839. MR2888306

[23] Zhi Zhong Sun, A second-order accurate linearized difference scheme for the two-dimensional Cahn-Hilliard equation, Math. Comp. 64 (1995), no. 212, 1463-1471, DOI 10.2307/2153365. MR.1308465 (96a:65133)

[24] Zhi-Zhong Sun and Qi-Ding Zhu, On Tsertsvadze's difference scheme for the KuramotoTsuzuki equation, J. Comput. Appl. Math. 98 (1998), no. 2, 289-304, DOI 10.1016/S03770427(98)00135-6. MR 1657022 (99m:65167)

[25] Cheng Wang, Xiaoming Wang, and Steven M. Wise, Unconditionally stable schemes for equations of thin film epitaxy, Discrete Contin. Dyn. Syst. 28 (2010), no. 1, 405-423, DOI 10.3934/dcds.2010.28.405. MR2629487(2011c:65186)

[26] S. M. Wise, C. Wang, and J. S. Lowengrub, An energy-stable and convergent finite-difference scheme for the phase field crystal equation, SIAM J. Numer. Anal. 47 (2009), no. 3, 22692288, DOI 10.1137/080738143. MR2519603 (2010f:65169)

[27] Chuanju $\mathrm{Xu}$ and Tao Tang, Stability analysis of large time-stepping methods for epitaxial growth models, SIAM J. Numer. Anal. 44 (2006), no. 4, 1759-1779, DOI 10.1137/050628143. MR:2257126(2007m:65078)

[28] Zhengru Zhang and Zhonghua Qiao, An adaptive time-stepping strategy for the Cahn-Hilliard equation, Commun. Comput. Phys. 11 (2012), no. 4, 1261-1278, DOI 10.4208/cicp.300810.140411s. MR2864085

[29] Yu Lin Zhou, Applications of Discrete Functional Analysis to the Finite Difference Method, International Academic Publishers, Beijing, 1991. MR.1133399 (92m:65001)

Department of Applied Mathematics, The Hong Kong Polytechnic University, Hung Hom, Kowloon, Hong Kong.

E-mail address: zqiao@polyu.edu.hk

Department of Mathematics, Southeast University, Nanjing, 210096, People's RepubLIC OF CHINA.

E-mail address: zzsun@seu.edu.cn

Laboratory of Mathematics and Complex Systems, Ministry of Education and School of Mathematical Sciences, Beijing Normal University, Beijing, 100875, People's RepubLIC OF CHINA.

E-mail address: zrzhang@bnu.edu.cn 